\title{
CARACTERÍSTICAS VOCAIS ACÚSTICAS DO SOM BASAL EM HOMENS COM FISSURA PÓS-FORAME REPARADA
}

\author{
Acoustic characteristics of vocal fry \\ in men with repaired post-foramen cleft
}

\author{
Giseane Conterno (1), Carla Aparecida Cielo (2), Vanessa Santos Elias ${ }^{(3)}$
}

\begin{abstract}
RESUMO
Tema: características vocais acústicas da emissão em registro basal de pacientes adultos masculinos, portadores de fissura palatina pós-forame reparada cirurgicamente. Procedimentos: estudo de casos envolvendo quatro sujeitos do sexo masculino portadores de fissura palatina pós-forame reparada cirurgicamente. Foi realizada análise acústica da emissão sustentada da vogal [a] em registro modal e basal, através dos programas Multi Dimensional Voice Program (análise da fonte glótica) e Real-Time Spectrogram (análise espectrográfica), ambos da Kay Elemetrics Corp. Resultados: por meio da análise espectrográfica do basal, observou-se: diminuição de definição, intensidade e de largura de banda de alguns formantes, diminuição da intensidade de todo o espectro, e discreta diminuição do ruído nas altas frequências; o efeito damping apresentou discreto aumento. Pela análise da fonte glótica no basal através do MDVPA, observou-se: frequência fundamental dentro da faixa do registro modal de fala e aumentada quando comparada à emissão para comparação em registro modal; grande aumento das medidas que evidenciam variação de frequência e de amplitude; maioria das medidas de jitter e de shimmer significativamente aumentadas; medidas de ruído, de quebras de voz, de segmentos não sonorizados e de tremor aumentadas; e índice de fonação suave (SPI) significativamente diminuído. Conclusão: os resultados evidenciam a alta instabilidade e ruído da emissão em registro basal, bem como sua fraca intensidade e diminuição do fluxo aéreo transglótico.
\end{abstract}

DESCRITORES: Fonoterapia; Voz; Fissura palatina; Espectrografia

\section{INTRODUÇÃO}

A fissura lábio-palatina é uma malformação congênita ${ }^{1,2}$ caracterizada por alteração na fusão dos processos faciais embrionários responsáveis pela formação da face, podendo acometer o lábio, o rebordo alveolar, o palato duro e o palato mole ${ }^{3}$.

Os indivíduos portadores desse tipo de malformação podem apresentar diversos acometimentos

(1) Fonoaudióloga; Mestre em Distúrbios da Comunicação Humana pela Universidade Federal de Santa Maria.

(2) Fonoaudióloga; Professora Adjunta do Departamento de Fonoaudiologia da Universidade Federal de Santa Maria, UFSM, Santa Maria, RS; Doutora em Linguagem Aplicada pela Pontifícia Universidade Católica do Rio Grande do Sul.

(3) Fonoaudióloga; Professora Adjunta do Departamento de Fonoaudiologia da Feevale, Novo Hamburgo, RS; Mestre em Distúrbios da Comunicação Humana pela Universidade Federal de Santa Maria.

Conflito de interesses: inexistente consequentes, contudo, uma das alterações que mais chama a atenção em pacientes fissurados, por afetar a comunicação, é a hipernasalidade. Esta decorre do fechamento inadequado do esfíncter velofaríngeo (EVF), o que acaba por provocar a falta de isolamento entre as cavidades oral e nasal ${ }^{3,4}$, contaminando assim os sons orais por um foco de ressonância nasal ${ }^{5}$, além de dificultar que o indivíduo mantenha a pressão de ar na cavidade oral para a produção da fala ${ }^{6}$. A voz hipernasal pode transmitir a impressão de limitações físicas e intelectuais e até mesmo alterações emocionais ao indivíduo que a apresenta ${ }^{5}$.

Uma das formas de tratamento da hipernasalidade é através de cirurgia corretiva ${ }^{7,8}$. Porém, muitas vezes, mesmo com a cirurgia reparadora, alguns fatores de ordem funcional conservam-se alterados. Para esses pacientes, a reabilitação fonoaudiológica, por meio da utilização de técnicas vocais é indicada ${ }^{8}$. 
O registro basal foi introduzido como técnica terapêutica por Boone e McFarlane em 1984. Este é produzido principalmente pela contração do músculo tireoaritenóideo que, por promover o encurtamento e a adução das pregas vocais, pode deixar a mucosa mais solta, a borda livre mais arredondada e o nível vertical da prega vocal mais rebaixado ${ }^{9,10}$. Durante a emissão vocal neste registro, o véu palatino encontra-se firmemente aproximado da parede faríngea posterior, o que pode levar à redução da hipernasalidade encontrada principalmente nas alterações o EVF ${ }^{11}$. Outros autores ${ }^{6,11-14}$ concordam que a utilização dessa técnica pode auxiliar o fechamento do EVF e, por conseguinte, diminuir a ressonância nasal, tão prejudicial à comunicação dos pacientes fissurados.

$\mathrm{Na}$ literatura, encontram-se estudos que descrevem o som basal como o registro vocal que apresenta maior componente de ruído na proporção harmônico-ruído (PHR) ${ }^{5,9}$. Além disso, os valores de jitter e de shimmer são significativamente maiores no som basal, quando comparados com o registro modal, sendo que o registro basal apresenta o fluxo aéreo, durante a produção sustentada de uma vogal, três vezes menor do que o fluxo aéreo durante a produção sustentada da mesma vogal em registro modal ${ }^{9}$.

São poucos os estudos da literatura atual que descrevem as características vocais acústicas do som basal. Desse modo, o presente trabalho tem como objetivo descrever as características vocais acústicas da emissão em registro basal de pacientes masculinos portadores de fissura palatina pósforame, reparada cirurgicamente.

\section{APRESENTAÇÃO DOS CASOS}

Estudo exploratório, retrospectivo e transversal, no qual foi utilizado o método de Estudo de Casos.

Os dados de todos os sujeitos que participaram da pesquisa foram obtidos do Banco de Dados do Laboratório de Voz da instituição de origem, sendo que os sujeitos passaram previamente pelos procedimentos descritos a seguir.

Foram cumpridas as determinações da norma 196/96 da Comissão Nacional de Ética em Pesquisa (CONEP), levando-se em consideração as preocupações éticas decorrentes da realização de experimentos e avaliações com seres humanos, de forma que os sujeitos foram avaliados somente após assinatura do Termo de Consentimento Livre e Esclarecido (TCLE) prévio.

Participou deste estudo um grupo composto por quatro indivíduos do sexo masculino, voluntários, que se enquadraram nos seguintes critérios de inclusão: ser esclarecido sobre a pesquisa e assinar o TCLE; ser portador de fissura palatina pós-forame, reparada cirurgicamente; ser do sexo masculino; ser adulto jovem, em função da influência das alterações hormonais e estruturais do enveIhecimento que podem causar a presbifonia ${ }^{15} \mathrm{e}$ do período da muda vocal sobre o aparelho fonador que, no homem, normalmente acontece entre os 13 e os 15 anos de idade ${ }^{16}$; e não ter realizado tratamento fonoaudiológico prévio, com o objetivo de evitar que compensações musculares adequadas, através do treinamento com técnicas vocais, tenham sido utilizadas para estimular o fechamento do EVF.

Os critérios de exclusão foram: não apresentar habilidade de realização da técnica do som basal, levando-se em consideração que nem todos os pacientes conseguem realizar todas as técnicas propostas; e possuir alterações auditivas, uma vez que a discriminação e o feedback auditivo têm um importante papel no controle da produção vocal ${ }^{17}$, influenciando o auto-monitoramento vocal ${ }^{18}$.

Para a realização do processo de seleção dos sujeitos e da coleta de dados foram utilizados os seguintes materiais: Termo de Autorização Institucional; TCLE; protocolo de anamnese; avaliação auditiva, que cada sujeito realizou por conta própria, por meio de encaminhamento ao médico otorrinolaringologista; e aparelho de minidisc $M Z R-70$, conectado a um microfone profissional MP- 68, unidirecional, com condensador a eletreto, da marca Le Son®.

Inicialmente, os voluntários responderam a uma anamnese para levantamento de dados de identificação, bem como de seu histórico, idade, diagnóstico ao nascimento, dados referentes à cirurgia, à realização ou não de fonoterapia prévia, dentre outros aspectos, com a finalidade de selecionar o grupo de estudo por meio dos critérios de inclusão e de exclusão.

Em seguida, os sujeitos participaram de uma explanação oral a respeito da importância do estudo em questão, dos critérios de inclusão e de exclusão, da importância da assinatura do TCLE e da técnica terapêutica do som basal. Nessa mesma ocasião, com o monitoramento de fonoaudiólogo, foram ensinados a executar a técnica do som basal para avaliação da habilidade na realização desta e para garantir que a executassem corretamente durante a coleta dos dados.

Os sujeitos trouxeram uma cópia da avaliação auditiva, previamente solicitada pelo médico otorrinolaringologista, em virtude dos critérios de exclusão.

Por meio dessa seleção, dos 14 voluntários a participar do estudo (cinco do sexo masculino e nove do sexo feminino), 10 foram descartados por 
não se adequarem aos critérios de inclusão e/ou exclusão da pesquisa, sendo o grupo de estudo composto de quatro indivíduos, voluntários, todos do sexo masculino, com idades entre 19 e 26 anos.

A coleta de dados se deu em dois momentos. No primeiro, os indivíduos foram orientados a emitir a vogal [a] ${ }^{19}$ sustentada no registro modal, em tom e intensidade habituais em tempo máximo de fonação ${ }^{18,20,21}$. No segundo, os participantes realizaram emissão em som basal em tempo máximo de fonação.

No momento das emissões, as vozes foram registradas em minidisc, posicionando-se o microfone a uma distância de cinco centímetros em frente à boca do sujeito ${ }^{5,21}$ e com um ângulo de $90^{\circ}$, para evitar interferências no sinal ${ }^{5}$.

A partir das etapas descritas anteriormente, realizadas com os sujeitos e cujos dados estavam armazenados no Banco de Dados, iniciaram-se os procedimentos do presente trabalho.

Foi realizada a análise acústica das emissões, utilizando-se o Multi Dimensional Voice Program Advanced (MDVPA) e o programa Real-Time Spectrogram, ambos da Kay Elemetrics Corp.

Deve-se ressaltar que as vozes foram editadas, visto que, segundo alguns autore ${ }^{4,19,20}$, as extremidades das emissões podem apresentar instabilidades fonatórias. Assim, foi eliminada a parte inicial e final de cada emissão vocal.

Apesar das vogais /i/ e / $\mathrm{u} /$ serem consideradas as vogais mais orais, a vogal /a/ foi utilizada nesta pesquisa em função de que o programa MDVPA a preconiza para análise. Ainda, tem-se conhecimento que é a vogal /a/ a mais utilizada em pesquisas em voz, tanto nacionais quanto internacionais, e também com outros programas de análise acústica.

A análise do espectrograma - em filtros de banda estreita e de banda larga - foi realizada, individualmente, por três fonoaudiólogas com experiência na área de acústica de voz, considerandose, para fins de resultados, a opinião em comum, ou a predominante, entre os dados do julgamento das avaliadoras, em relação às características dos traçados espectrográficos. Para aumentar a confiabilidade dos resultados, as espectrografias antes e durante o som basal de cada sujeito apareceram duas vezes aleatoriamente para as juízas, de modo que cada juíza avaliou duas vezes a mesma espectrografia sem saber. Estas receberam como informação somente quais espectrografias correspondiam ao registro modal e quais ao registro basal, porém, sem especificar quais correspondiam ao mesmo sujeito, dessa forma, cada espectrografia foi avaliada isoladamente. Uma quarta juíza foi solicitada nos casos de empate entre o julgamento das três primeiras juízas.
Foi utilizado um protocolo de avaliação espectrográfica, sendo os itens avaliados: intensidade dos formantes; intensidade das altas frequências; intensidade em todo o espectro vocal; presença de ruído; largura de banda do formante; definição dos formantes; definição de harmônicos; regularidade do traçado; e anti-ressonância/damping em todo o espectro vocal. Salienta-se que as juizas não conheciam o objetivo da pesquisa, garantindo maior confiabilidade de suas avaliações.

Para a análise acústica da voz no Multi Dimensional Voice Program Advanced (MDVPA), não houve necessidade de juizes, pois o programa fornece resultados automáticos para cada medida, conforme o sexo dos sujeitos. Somente para a faixa de normalidade da f0 optou-se por utilizar a faixa de normalidade que, nos homens, vai de $80 \mathrm{a} 150 \mathrm{~Hz}^{5}$.

Os itens avaliados foram: Frequência fundamental média (f0); Frequência fundamental mais alta (fhi); Frequência fundamental mais baixa (flo); Desvio-padrão da frequência fundamental (STD); Variação da frequência fundamental fonatória (PFR); Jitter absoluto (Jita); Jitter percentual (Jitt); Quociente de perturbação do pitch (PPQ); Coeficiente da variação da frequência fundamental (vf0); Shimmer em dB (ShdB); Shimmer percentual (Shim); Quociente de perturbação da amplitude (APQ); Coeficiente da variação da amplitude (vAm); Proporção ruído-harmônico (NHR); Índice de turbulência da voz (VTI); Índice de fonação suave (SPI); Grau de quebra da voz (DVB); Grau dos componentes sub-harmônicos (DSH); Grau de silêncio período sem voz (DUV); Número de quebras vocais (NVB); Número de segmentos sub-harmônicos (NSH); Número de segmentos não sonorizados (NUV); Frequência da frequência do tremor (Fftr); Frequência da amplitude do tremor (Fatr); Índice de severidade da frequência do tremor (FTRI); Índice de severidade da amplitude do tremor (ATRI); Quociente de perturbação do pitch suavizado (SPPQ); Quociente de perturbação da amplitude suavizado (sAPQ).

Deve-se ressaltar que o National Center for Voice and Speech (NCVS) classifica como uma voz do tipo 3 as vozes caóticas ou aleatórias, em função da grande aperiodicidade apresentada, afirmando que elas são melhor analisadas com espectrografia e perceptivamente, não sendo seguramente analisadas pelo MDVPA, que pode não conseguir extrair as medidas. $\mathrm{O}$ som basal poderia se encaixar nessa classificação devido a sua aperiodicidade audível. No entanto, nesta pesquisa, o MDVPA conseguiu extrair as medidas acústicas das emissões analisadas (forneceu o diagrama radial e os valores numéricos). 
A pesquisa foi previamente aprovada pelo Comitê de Ética em Pesquisa da instituição de origem (protocolo número 23081.008439/2007-16).

Os dados encontrados para as espectrografias foram analisados de forma qualitativa.

Para testar se existia diferença significante entre as médias Modal e Basal e a Normalidade, para os resultados obtidos por meio do programa MDVPA, utilizou-se o teste t de Student, empregado quando se deseja comparar a média de uma amostra com um valor de referência. Assim, compararam-se os valores de $t$ calculados a partir da amostra com o valor de t tabelado. Para isso, adotou-se um nível de significância $(\alpha)$ igual a $5 \%$.

Para verificar a significância das variações entre as médias do registro Modal e as médias do registro Basal também foi empregado o teste t de Student, ao nível de $5 \%$ de significância $(\alpha=0,05)$. Somente nos casos das variáveis DSH e NSH não foi possível aplicar o teste t de Student porque todos os valores eram nulos.

\section{RESULTADOS}

Os resultados da avaliação vocal dos sujeitos, por meio das espectrografias de banda larga e de banda estreita, estão expostos nas Tabelas 1 e 2 .

Os resultados da avaliação vocal acústica dos sujeitos, por meio do MDVPA, estão expostos nas Tabelas 3, 4 e 5 .

\section{DISCUSSÃO}

Quanto à análise do filtro vocal, por meio de espectrografias, antes e durante o som basal em fissurados masculinos, observou-se que a intensidade dos formantes $(F)$, das altas frequências e de todo o espectro vocal, de forma geral, tanto na espectrografia de banda larga (EBL) quanto na espectrografia de banda estreita (EBE), permaneceu igual ou diminuiu durante o som basal, aparecendo na maioria dos casos como fraca (Tabelas 1 e 2), com exceção do sujeito 1, na EBL, que apresentou aumento da intensidade de F1, e do sujeito 4, na EBE, que apresentou aumento da intensidade em todo o espectro vocal durante a emissão em registro basal. Além disso, o damping aumentou durante o basal.

Tais achados vão ao encontro de um estudo ${ }^{22}$ que afirma que a redução da intensidade do formante, a redução da intensidade das altas frequências, incluindo o terceiro formante, e a redução da intensidade em todo o espectro vocal são, dentre outras, as características do espectro acústico da nasalidade. Outros autores ${ }^{23,24}$ também observaram em seus trabalhos realizados, comparando-se vogais nasalizadas com vogais orais, que uma das características das vogais nasalizadas é a reduzida intensidade dos F. De acordo com os mesmos autores, essa redução de intensidade se deve à adição das características de amortecimento das superfícies da cavidade nasal, devido às antirressonâncias e aos $F$ nasais, que podem retirar energia acústica dos $\mathrm{F}$ vizinhos. Ainda, determinado estudo ${ }^{4}$ afirma que o efeito de incorporar o acoplamento nasal ao oral pode alargar e suavizar os picos no espectro vocálico.

Os estudos mencionados se referem ao contraste entre emissões orais e nasais em registro modal, mas, no presente estudo, trata-se de emissões nasais em registro modal e em registro basal, verificando-se que a nasalidade em si manifestou-se, conforme a literatura referida, diminuindo a intensidade da ressonância e evidenciando damping também em registro basal.

Dessa forma, diferente do esperado, levandose em consideração o consenso entre vários autores ${ }^{6,11-14}$ de que a utilização da técnica do som basal auxilia o fechamento do EVF e, consequentemente, diminui a ressonância nasal, a antirressonância permaneceu presente na maioria dos sujeitos e, em um caso, aumentou (sujeito 3), mesmo durante a produção do som basal.

$\mathrm{Na}$ emissão em registro basal, encontra-se uma intensidade fonatória bastante débil, com corrente aérea entre as pregas vocais mínima ${ }^{9,25}$, diminuição da estabilidade fonatória nos domínios da frequência e amplitude ${ }^{9}$, além de frequência fundamental muito baixa ${ }^{26}$, fatores que poderiam justificar a fraca intensidade e a diminuição da definição dos $\mathrm{F}$ indicadas pela espectrografia durante a emissão em registro basal (Tabela 1).

A presença de ruído no espectro manifestou-se com a diminuição discreta da definição da largura de banda de F3 e F4, na EBL, evidenciando a aperiodicidade característica da emissão em basal ${ }^{5}$.

No entanto, houve diminuição do ruído nas altas frequências na $E B E$, possivelmente pela falta de energia/intensidade nessas frequências. Isso pode se justificar pelo fato de o som basal produzir as frequências mais graves da tessitura da voz, apresentar intensidade diminuída e pouca energia nas frequências agudas ${ }^{9,25}$.

Acredita-se, também, que a presença de ruído observada durante a emissão em registro basal seja consequência da hipernasalidade característica da fala de pacientes portadores de fissura palatina, quando as ondas sonoras que carregam a vibração da laringe são amortecidas dentro da cavidade nasal ${ }^{3,27}$, gerando "apagamentos" (efeito damping) no espectro ${ }^{19}$, o que se verificou neste estudo em que houve aumento discreto de damping na EBL, bem como diminuição geral de intensidade e de definição de $F$ (Tabela 1). 

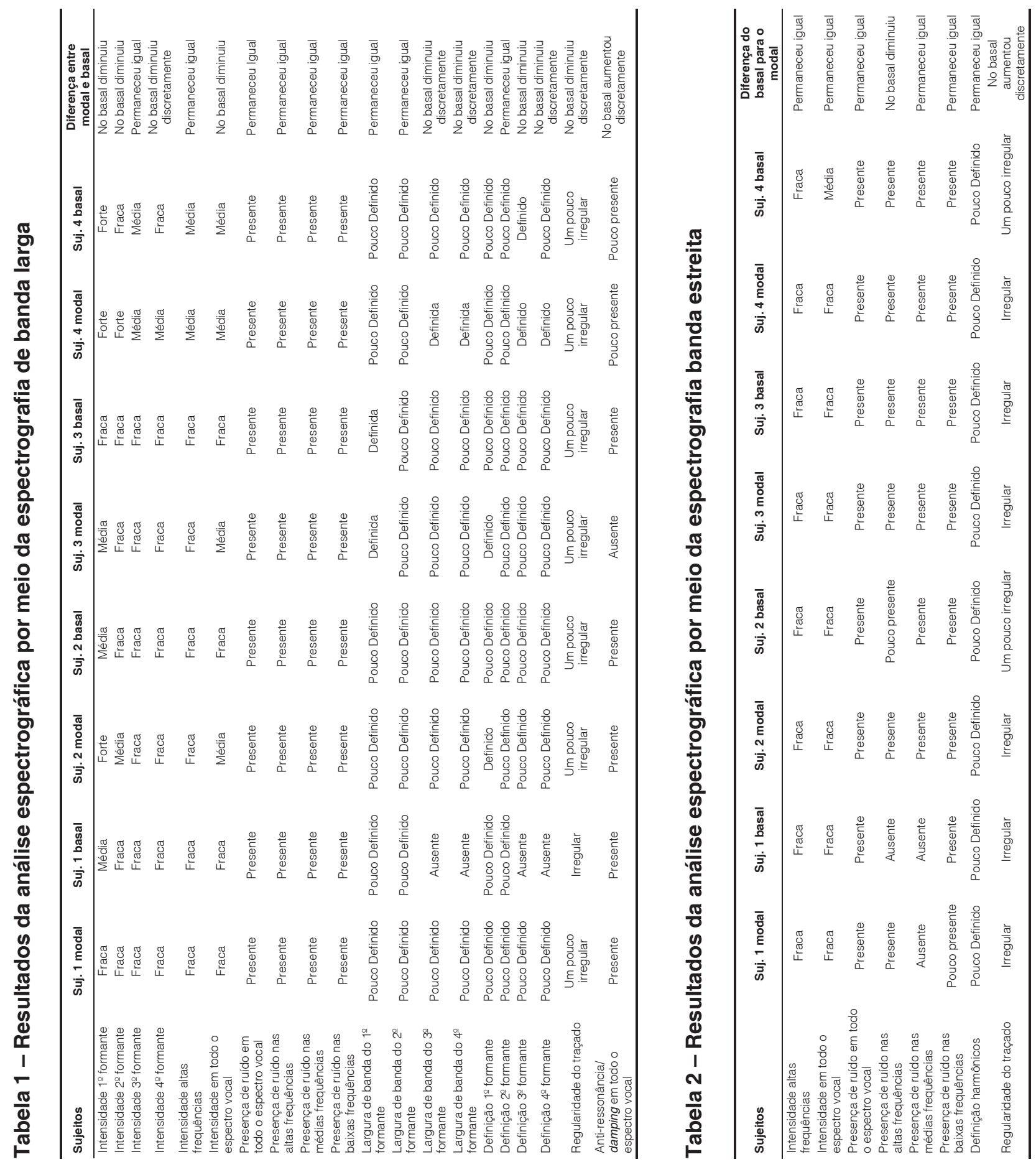
Tabela 3 - Resultado das medidas de frequência fundamental, por meio do programa MDVPA

\begin{tabular}{ccccc}
\hline Variáveis & Média Registro modal & Média Registro basal & Variação & MDVPA padrão \\
\hline f0 $(\mathrm{Hz})$ & 116.668 & 129.309 & +12.641 & $80-150^{\star *}$ \\
fhi $(\mathrm{Hz})$ & 119.876 & 242.016 & +122.14 & 150.080 \\
flo $(\mathrm{Hz})$ & 113.492 & 72.091 & $-41.401^{*}$ & 140.418 \\
STD $(\mathrm{Hz})$ & 1.429 & 42.962 & $+41.0007480)$ & 1.349 \\
PRF & 2 & 17.75 & $+15.75 *(p=0,091040)$ & 2.095 \\
\hline
\end{tabular}

* variação estatisticamente significante

** faixa adotada neste estudo conforme Behlau et al. (2001)

Teste t de Student, ao nível de 5\% de significância.

Tabela 4 - Resultado das medidas de perturbação da frequência e intensidade, por meio do programa MDVPA

\begin{tabular}{|c|c|c|c|c|}
\hline Variáveis & Média Registro modal & Média Registro basal & Variação & MDVPA padrão \\
\hline Jita $\mu \mathrm{s}$ & 71.602 & $737.747^{* \star *}(p=0,031961)$ & $\begin{array}{c}+666.145^{*} \\
(p=0,037842)\end{array}$ & 83.20 \\
\hline Jitt $\%$ & 0.824 & $7.349^{\star \star *}(p=0,029814)$ & $+6.525^{*}(p=0,039751)$ & 1.040 \\
\hline PPQ \% & 0.491 & $4.524^{\star \star *}(p=0,011443)$ & $+4.033^{*}(p=0,014806)$ & 0.840 \\
\hline SPPQ \% & 0.390 & 13.205 & +12.815 & 1.020 \\
\hline vfo \% & $1.246^{* \star}(p=0,033041)$ & 24.645 & +23.399 & 1.100 \\
\hline ShdB (dB) & $0.532^{* \star}(p=0,008743)$ & $2.413^{* * *}(p=0,040727)$ & +1.881 & 0.350 \\
\hline Shim \% & $6.113^{* \star}(p=0,008746)$ & $20.054^{\star * *}(p=0,008771)$ & $\begin{array}{c}+13.941 \text { * } \\
(p=0,041048)\end{array}$ & 3.810 \\
\hline APQ \% & $4.992^{* \star}(p=0,012330)$ & $17.062^{\star \star \star}(p=0,007072)$ & $+12.07^{*}(p=0,012319)$ & 3.070 \\
\hline SAPQ \% & 4.175 & $22.609^{* * *}(\mathrm{p}=0,000036)$ & +18.434 & 4.230 \\
\hline vAm \% & $15.989^{* *}(p=0,014167)$ & $38.357^{* * *}(p=0,011790)$ & +22.368 & 8.200 \\
\hline
\end{tabular}

* variação estatisticamente significante

** diferença estatisticamente significante entre a média modal e o MDVPA padrão

*** diferença estatisticamente significante entre a média basal e o MDVPA padrão

Teste t de Student, ao nível de 5\% de significância.

Tabela 5 - Resultado das medidas de ruído, de quebra de voz, de segmentos não sonorizados, de sub-harmônicos e de tremor, por meio do programa MDVPA

\begin{tabular}{|c|c|c|c|c|}
\hline Variáveis & Média Registro modal & Média Registro basal & Variação & MDVPA padrão \\
\hline $\mathrm{NHR}$ & 0.160 & $0.505^{* *}(p=0,006293)$ & $+0.345^{*}(p=0,007276)$ & 0.190 \\
\hline VTI & 0.063 & 0.085 & +0.022 & 0.061 \\
\hline SPI & 16.421 & 11.906 & $-4.515^{*}(p=0,042689)$ & 14.120 \\
\hline DVB \% & 0.000 & 54.918 & $+54.918 *(p=0,061329)$ & 1.000 \\
\hline DSH \% & 0.000 & 0.000 & --- & 1.000 \\
\hline DUV \% & 0.5 & $72.475^{* *}(p=0,004949)$ & $+71.975 *(p=0,005432)$ & 1.000 \\
\hline NVB & 0 & 3.25 & +3.25 & 0.900 \\
\hline $\mathrm{NSH}$ & 0 & 0 & --- & 0.900 \\
\hline NUV & 0.25 & $71^{* *}(p=0,046770)$ & $+70.75^{*}(p=0,046503)$ & 0.900 \\
\hline $\mathrm{Fftr}(\mathrm{Hz})$ & 0 & $8.733^{* *}(p=0,000671)$ & $+8.733 *(p=0,000133)$ & 3.655 \\
\hline Fatr $(\mathrm{Hz})$ & 0 & 5.415 & $+5.415^{*}(p=0,088023)$ & 2.728 \\
\hline FTRI \% & 0 & 10.837 & +10.837 & 0.950 \\
\hline ATRI \% & 0 & 7.009 & +7.009 & 4.370 \\
\hline
\end{tabular}

* variação estatisticamente significante

** diferença estatisticamente significante entre a média basal e o MDVPA padrão

Teste t de Student, ao nível de 5\% de significância. 
Ainda, em relação ao $\mathrm{F} 1$, que é o mais grave, nos sujeitos 2 e 3 , a definição também diminuiu, e isso se deve ao fato de o som basal ser uma emissão bastante crepitante e aperiódica ${ }^{5}$.

Na emissão em registro modal, anterior ao basal, os harmônicos apresentaram-se pouco definidos em função da presença da hipernasalidade, visto que a voz com ressonância nasal apresenta no espectro vocal presença de harmônicos reforçados nas frequências em que a energia não é normalmente esperada ${ }^{23}$. Essas frequências reforçadas, que constituem ressonâncias extras, podem ser consideradas as ressonâncias da cavidade nasal. Determinado autor ${ }^{4}$ refere que, no espectro das vogais nasais, observou-se, dentre outras características, redução da intensidade dos harmônicos de alta frequência, o que ocorreu neste estudo também na emissão em basal, provavelmente decorrente da presença de fissura reparada nos sujeitos.

Ainda, em um estudo que teve como objetivo examinar a qualidade vocal e investigar os efeitos do gênero na qualidade vocal de 28 crianças com fissura palatina uni ou bilateral ${ }^{28}$, foram encontrados resultados que levaram a concluir que a fonação dos sujeitos fissurados masculinos é associada com rouquidão e aspereza. $E$ tem-se conhecimento de que, na análise espectrográfica das vozes roucas, os harmônicos apresentam-se em menor número e com um traçado menos definido, com instabilidade e baixa amplitude devido à presença de ruído e talvez da variação da f0 ${ }^{29}$.

Possivelmente, no registro basal, os harmônicos permaneceram pouco definidos e com discreta diminuição da regularidade em função da característica crepitante e irregular do som basal ${ }^{5,9,30,31}$, da hipernasalidade já mencionada e de sua característica de menor intensidade de emissão ${ }^{9,25}$.

O que não se justifica é o fato de as emissões prévias em registro modal terem sido mais intensas, quando o que se esperava era que o som basal diminuísse a abertura do EVF e proporcionasse maior oralidade à emissão como se afirma em outros trabalhos ${ }^{6,11-14}$.

Quanto à análise da fonte vocal, por meio do programa MDVPA, antes e durante o som basal em fissurados, observou-se que a média da frequência fundamental, definida como o número de vibrações por segundo produzidas pelas pregas vocais ${ }^{5,20,21}$, em registro basal, ficou dentro da faixa esperada para registro modal de fala e ainda apresentou aumento quando comparada ao registro modal (Tabela 3), o que vai de encontro à literatura que afirma que o som basal apresenta frequência fundamental muito grave, na faixa de 20 a $70 \mathrm{~Hz}{ }^{5,9,26}$.
Como a f0 é o resultado da interação entre o comprimento, a massa e a tensão das pregas vocais durante a fonação ${ }^{5,21}$, é natural que o som basal apresente uma frequência fundamental mais baixa que os outros registros já que, sua produção se dá principalmente pela contração do músculo tireoaritenoideo, que promove o encurtamento e a adução das pregas vocais, deixando a mucosa mais solta, a borda mais arredondada e as pregas vocais mais rebaixadas e espessas ${ }^{9-11,32}$.

É possível que, mesmo tendo sido orientado quanto à execução do som basal, os sujeitos tenham realizado o vocal fry tenso com laringe alta ${ }^{11}$ o qual pode alcançar frequências mais agudas, tendo sido mascarado pela presença da nasalidade.

Apesar de determinados autores ${ }^{28}$ afirmarem que é possível observar uma f0 significativamente mais baixa em sujeitos com fissura palatina, quando comparados a sujeitos normais, como estratégia utilizada para diminuir a hipernasalidade vocal, nesta pesquisa isso não ocorreu, visto que, no registro modal, a f0 ficou dentro da faixa esperada para homens (Tabela 3 ), apesar da presença da fissura reparada.

$\mathrm{Na}$ emissão em registro basal, verificou-se grande variação das medidas de vf0 (Tabela 4), STD, fhi, flo e PFR, as duas últimas com significância estatística (Tabela 3), estando todas acima do esperado pelo MDVPA, com exceção da flo, que evidenciou o alcance do registro basal ao chegar na faixa dos $70 \mathrm{~Hz}$ (Tabela 3). Todas essas medidas evidenciam a alta instabilidade da emissão em registro basal quando comparado ao modal e ao esperado pelo MDVPA.

$O$ registro basal apresenta periodicidade perturbada, com excitação glótica irregular ${ }^{31}$, já que é geralmente produzido com múltiplos pulsos vocais, com um curto período de fase aberta e um longo período de fase fechada, apresentando as margens das pregas vocais mais flácidas, diminuição do fluxo aéreo e aumento da resistência glótica, além da própria diminuição da estabilidade fonatória nos domínios da frequência e amplitude e aumento da energia ruidosa ${ }^{9,30,33}$.

Todas as medidas de jitter, definido como variabilidade da frequência fundamental ciclo a ciclo ${ }^{5,20}$, apresentaram-se significativamente aumentadas no registro basal quando comparadas ao registro modal e ao MDVPA, com exceção do SPPQ e da vf0, que estavam aumentadas sem significância estatística (Tabela 4). Resultados que vão ao encontro de um estudo ${ }^{9}$ que encontrou valores de jitter significativamente maiores no registro basal quando comparado com o registro modal.

Como essas medidas evidenciam a irregularidade da vibração da mucosa das pregas vocais, 
além de serem correlacionadas com as características biomecânicas das pregas vocais e com a variação do controle neuromuscular ${ }^{20}$, podese apontar novamente a aperiodicidade do som basal ${ }^{9,30,31}$ e, principalmente o fato de a produção desse registro se dar com a fase fechada do ciclo vibratório muito longa em relação à extensão do período inteiro ${ }^{9,33}$, como causa dos valores altos dessas medidas, já que o aumento de jitter no registro basal indica diminuição da estabilidade fonatória nos domínios da frequência ${ }^{9}$, o que se traduz à oreIha humana como ruído à emissão.

No registro modal, todas as medidas de jitter ficaram dentro do esperado pelo MDVPA, com exceção da vfO aumentada, sugerindo dificuldades na estabilidade da emissão sustentada. No entanto, essa medida isolada deve ser observada com cautela, uma vez que a diferença entre fhi e flo, e a PFR ficaram dentro do esperado sugerindo estabilidade (Tabela 4).

Todas as medidas de Shimmer, definido como a perturbação ou variabilidade da amplitude ciclo a ciclo ${ }^{5,20}$, apresentaram-se significativamente maiores do que o esperado pelo MDVPA, tanto em registro modal (excetuando-se o SAPQ) quanto em basal, sugerindo a presença de ruído em ambos os registros, podendo haver relação com a hipernasalidade dos sujeitos.

No entanto, em registro basal, todas as medidas de shimmer foram maiores do que no registro modal, sendo que as variações do Shim e do APQ foram estatisticamente significantes (Tabela 4).

Essas medidas aumentadas de Shimmer indicam maior perturbação fonatória nos domínios da amplitude ${ }^{9} \mathrm{e}$ podem estar correlacionadas, principalmente, com o fato de o fluxo aéreo no registro basal ser diminuído e apresentar um curto período com as pregas vocais abertas e um longo período com as pregas vocais fechadas ${ }^{9,33}$.

Além disso, o valor de shimmer tende a ser elevado nas frequências graves e de baixa intensidade ${ }^{5}$, e seus valores crescem quanto maior a quantidade de ruído numa emissão, características presentes na emissão em registro basal 5,9,26.

Ainda, pode-se inferir que a falta de prática dos pacientes participantes da pesquisa em utilizarem esse tipo de registro pode ter trazido como consequência um certo grau de incoordenação pneumofonoarticulatória, que pode ter influenciado os valores aumentados das medidas de shimmere de vAm (Tabela 4), bem como os de jitter e de vf0 (Tabela 4), como discutido anteriormente.

Dentre as medidas de ruído, o NRH em registro basal apresentou-se significativamente aumentado quando comparando ao registro modal e ao MDVPA (Tabela 5).
Esses dados vão ao encontro da literatura que aponta que o basal é o registro vocal que apresenta maior componente de ruído na proporção harmônico-ruído (PHR) ${ }^{5}$, provavelmente em função da excitação glótica irregular do som basal ${ }^{31}$ e da presença de crepitação, características deste registro ${ }^{5}$.

Tais afirmações são pertinentes ao se analisar determinado estudo ${ }^{9}$ que encontrou valores significativamente menores na relação sinal-ruído durante a emissão em registro basal quando comparada ao registro modal, o que corresponde ao elevado valor da relação ruído-harmônico encontrado neste estudo.

$\mathrm{O}$ valor do VTI, que corresponde ao índice de turbulência da voz ${ }^{20}$, em registro basal, também se apresentou aumentado quando comparado ao registro modal e ao padrão MDVPA (Tabela 5). O valor do SPI apresentou variação negativa estatisticamente significante em relação ao registro modal e também foi menor que o esperado pelo MDVPA (Tabela 5).

Esses valores aumentados de VTI, em relação ao esperado e ao modal, se devem possivelmente à presença de crepitação e irregularidade vibratória ao nível da glote durante o basal. Os valores diminuídos de SPI, em relação ao esperado e ao modal, relacionam-se ao modo como se dá a coaptação glótica durante o som basal: estreito fechamento das pregas vocais, fluxo aéreo diminuído, um longo período com as pregas vocais fechadas, em completa adução, e resistência glótica aumentada ${ }^{5,9,31,33}$, já que o aumento do valor de SPI é relacionado com vozes soprosas ${ }^{34}$, ou seja, que apresentam escape aéreo durante a fonação ${ }^{18}$.

Em registro modal, apenas as medidas de VTI e de SPI, relacionadas ao fluxo aéreo transglótico e à adução glótica, ficaram discretamente acima do esperado pelo MDVPA (Tabela 5), concordando com os aumentos encontrados nas medidas de shimmer já discutidas anteriormente (Tabela 4).

O modo de coaptação das pregas vocais durante o registro basal também justifica as medidas significativamente aumentadas de segmentos não sonorizados (NUV), em relação ao MDVPA e ao modal, e de quebra de voz aumentadas em relação ao MDVPA e ao modal, com significância no modal para DVB (Tabela 5).

De acordo com a literatura consultada ${ }^{20}$ medidas aumentadas de segmentos sub-harmônicos podem corresponder a qualidades vocais diplofônicas ou emissões em fry. As medidas de segmentos subharmônicos apresentaram-se nulas neste estudo, tanto em registro modal quanto em registro basal (Tabela 5), evidenciando a vibração exclusiva da fonte glótica em ambos os registros. 
Todas as medidas de tremor, relacionadas com a variação a longo termo da amplitude e frequência do sinal vocal ${ }^{20}$, principalmente as variações periódicas da voz, apresentaram-se aumentadas no registro basal quando comparadas ao MDVPA (Fftr aumentado significativamente) e ao modal (Fftr e Fatr aumentados significativamente) (Tabela 5).

De acordo com a literatura ${ }^{20} \mathrm{o}$ tremor vocal provém de um ou de vários componentes da fala, no nível respiratório, fonatório ou articulatório; no caso do registro basal, esse tremor caracterizado pelas medidas aumentadas seria proveniente do nível fonatório, visto que a emissão vocal durante a produção desse registro mostra crepitação e irregularidade vibratória ao nível da glote ${ }^{5,31}$.

\section{CONCLUSÃO}

Quanto à análise espectrográfica, durante o som basal em homens fissurados, concluiu-se que houve diminuição de definição, de intensidade e de definição de largura de banda de alguns $F$, bem como diminuição da intensidade de todo o espectro e discreta diminuição do ruído nas altas frequências. $\mathrm{O}$ efeito damping apresentou discreto aumento, e os harmônicos mantiveram-se pouco definidos em relação ao registro modal.
Quanto à análise da fonte vocal, durante o som basal em homens fissurados, concluiu-se que a f0 ficou dentro da faixa esperada para registro modal de fala e ainda apresentou aumento quando comparada ao registro modal, o que contraria a literatura.

Houve grande aumento das medidas de vfo, vAm, STD, fhi, intervalo entre fhi e flo e PFR, evidenciando a alta instabilidade da emissão em registro basal.

A maioria das medidas de jitter e de shimmer, em basal, mostraram-se significantemente aumentadas, evidenciando alta variabilidade de curto termo que pode se traduzir por aperiodicidade e ruído à emissão.

Todas as medidas de ruído, de quebras de voz, de segmentos não sonorizados e de tremor mostraram-se aumentadas no registro basal, evidenciando as características típicas do ciclo vibratório nesse registro; e o SPI foi diminuído em relação ao esperado e significativamente diminuído em comparação ao modal, provavelmente em função da maior adução glótica que ocorre no basal.

Ao contrário do esperado, as medidas de segmentos sub-harmônicos apresentaram-se nulas, tanto em registro modal quanto em registro basal, evidenciando a vibração exclusiva da fonte glótica em ambos os registros.

\begin{abstract}
Purpose: to describe the vocal acoustic characteristics of emission in vocal fry of male adult patients, with surgically repaired post-foramen cleft. Methods: study of cases involving four male subjects with surgically repaired post-foramen cleft. Acoustic analysis concerning sustained emission of vowel [a] was accomplished in modal register and vocal fry, through the programs Multi Dimensional Voice Program (glottic source analysis) and Real-Time Spectrogram (spectrographic analysis), both by Kay Elemetrics Corp. Results: through the vocal fry spectrography analysis we perceived: decrease of definition, intensity and definition band spectrography in some formants, decrease of intensity as for the entire spectrum, and a slight decrease of noise in high frequencies; damping effect showed slight increase. Through the glottic source analysis in vocal fry, we perceived: fundamental frequency in the modal speech register zone and it was higher when compared to the emission for comparison in the modal register; large increase in the measures that show variation of frequency and amplitude; most of the jitter and shimmer measures were significantly increased; increased measures of noise, voice break, unvoiced segment and vocal tremor; and soft phonation index (SPI) significantly decreased. Conclusions: the findings evidence the high instability and the emission noise in fry register, as well as its weak intensity and diminished transglottic air flow.
\end{abstract}

KEYWORDS: Speech Therapy; Voice; Cleft Palate; Spectrography 


\section{REFERÊNCIAS}

1. Ysunza A, Pamplona C. Disfunción del esfínter velofaríngeo y su tratamiento. Cir Plast. 2006; 16(1):62-8.

2. Sedano MR, Rodriguez AJG, Morovic ICG, Pizarro RO, Alarcón RJ, Salgado ME. Fisura labial y/o palatina en un centro de derivación de malformaciones congénitas. Rev Chil Ultrason. 2007; 10(1):4-10.

3. Matos HM. LA Articulación del habla en individuos con hendiduras labiopalatinas corregidas: estudio de dos casos. Rev. CEFAC. 2006; 8(2):186-97.

4. Souza MCQ. Características espectrais da nasalidade. [dissertação]. São Paulo (SP): Universidade de São Paulo; 2003.

5. Behlau M, Madazio G, Feijo D, Pontes P. Avaliação de voz. In: Behlau M. organizador. Voz: o livro do especialista. vol 1. Rio de Janeiro: Revinter; 2001. p. 85-172.

6. Elias VS. Eficácia do uso do som basal no fechamento do esfíncter velofaríngeo [dissertação]. Santa Maria (RS): Universidade Federal de Santa Maria; 2005.

7. Cheng N, Zhao M, Qi K, Deng H, Fang Z, Song R. A modified procedure for velopharyngeal sphincteroplasty in primary cleft palate repair and secondary velopharyngeal incompetence treatment and its preliminary results. J Plastic Reconstruct Aesthet Surg. 2006; 59(8):817-25.

8. Priester GH, Goorhuis-Brouwer SM. Speech and language development in toddlers with and without cleft palate. Int J Pediatr Otorhinolaryngol. 2008; 72(6):801-6.

9. Blomgren $\mathrm{M}$, Chen $\mathrm{Y}, \mathrm{Ng} \mathrm{ML}$, Gilbert HR. Acoustic, aerodynamic, physiologic, and perceptual properties of modal and vocal fry registers. J Acoust Soc Am. 1998; 103(5Pt1):2649-58.

10. Serrano DMS, Suehara $A B$, Fouquet $M L$, Gonçalves AJ. Uso do som crepitante grave (modelo vocal fry) nas laringectomias parciais verticais. Dist Comun. 2005; 17(1):19-25.

11. Pinho SMR. Terapia vocal. In: Pinho SMR. Tópicos em voz. Rio de Janeiro: Guanabara Koogan; 2001. p. 1-17.

12. Behlau M, Machado L, Guedes Z, Pontes P, Pontes A. Using vocal fry to trat nasality problems. In: Pacific Voice Conference. Tenth Annual, San Francisco, California, 1997.

13. Elias VS, Cielo CA, Knak IS, Faller GJ. Efeito do som basal em um caso de deficiência velofaríngea leve. In: XII Congresso Brasileiro de Fonoaudiologia, II Congresso Sulbrasileiro de Fonoaudiologia, 2004, Foz do Iguaçu. Anais. Foz do Iguaçu: Sociedade Brasileira de Fonoaudiologia, 2004.
14. Elias VS, Cielo CA. Utilização da técnica do som basal para estimular o fechamento do esfíncter velofaríngico de sujeito portadores de fissura palatina operada. Tema Livre. Congresso Brasileiro de Terapias em Fonoaudiologia, out, Porto Alegre, 2005.

15. Soares EB, Borba DT, Barbosa TK, Medved DM, Montenegro ACA. Hábitos vocais em dois grupos de idosos. Rev. CEFAC. 2007; 9(2):221-7. dx.doi. org/10.1590/S1516-18462007000200011

16. Santos MAO, Moura JMP, Duprat AC, Costa $\mathrm{HO}$, Azevedo BB. A interferência da muda vocal nas lesões estruturais das pregas vocais. Rev Bras Otorrinolaringol. 2007; 73(2):226-30.

17. Prado AC. Principais características da produção vocal do deficiente auditivo. Rev. CEFAC. 2007; 9(3):404-10. dx.doi.org/10.1590/ S1516-18462007000300014

18. Bolzan GP, Cielo CA, Brum DM. Efeitos do som basal em fendas glóticas. Rev. CEFAC. 2008; 10(2):218-25. dx.doi.org/10.1590/S151618462008000200011

19. Pinho SMR, Camargo Z. Introdução à análise acústica da voz e da fala. In: Pinho SMR. Tópicos em voz. Rio de Janeiro: Guanabara Koogan; 2001. p. $19-45$.

20. Barros APB, Carrara-De Angelis E. Análise acústica da voz. In: Dedivitis RA, Barros APB. Métodos de avaliação e diagnóstico de laringe e voz. São Paulo: Lovise; 2002. p. 201-21.

21. Felippe ACN, Grillo MHMM, Grechi TH. Normatização de medidas acústicas para vozes normais. Rev Bras Otorrinolaringol. 2006; 72(5):659-64.

22. Scalco MAG. O comportamento do primeiro formante e da largura de banda da nasal palatal em relação a variações de área e volume nasal. [dissertação]. Porto Alegre (RS): Pontifícia Universidade Católica do Rio Grande do Sul; 2002. 23. Schwartz MF. The acoustics of normal and nasal vowel production. Cleft Palate J. 1968; 5:125-40.

24. Behlau MS, Pontes PAL, Ganança MM, Tosi O. Análise espectrográfica de formantes das vogais do português brasileiro. Acta Awho. 1988; 7(2):74-85.

25. Boone DR, McFarlane SC. A voz e a terapia vocal. 5. ed. Porto Alegre: Artes Médicas; 2003.

26. Ishi CT, Sakakibara KI, Ishiguro H, Hagita N. A method for automatic detection of vocal fry. IEEE Transact Audio Speech Lang Process. 2008; 16(1):47-56.

27. Ysunza AR. Fisiología de músculos faríngeos posterior a la restauración quirúrgica del esfínter velofaríngeo. Gac Méd Méx. 2005; 141(3):195-9.

28. Van Lierde $K$, Claeys $S$, Bodt $M$, Van Cauwenberge P. Vocal quality characteristics 
in children with cleft palate: a multiparameter approach. J Voice. 2004; 18(3):354-62.

29. Pontes PAL, Vieira VP, Gonçalves MIR, Pontes AAL. Características das vozes roucas, ásperas e normais: análise acústica espectrográfica comparativa. Rev Bras Otorrinolaringol. 2002; 68(2):182-8.

30. Gendrot C, Henrich N, Sshade G, Muller F, Expert R. Vocal folds vibratory patterns of laryngeal mechanism M0 as investigated with high speed cinematography and electroglottography. In: International Conference on Voice Physiology and Biomechanics, Marseille, France, Aug. 2004.
31. Ishi CT. Analysis of autocorrelation-based parameters in Creaky voice. Acoust Sci Technol. 2004; 25(4):299-302.

32. Lacabe MIU, Gonzalez SF, Girbau MM, Sarrasqueta L, Urrutia RG. Voz cantada. Rev Med Univ Navarra. 2006; 50(3):49-55.

33. Slifka J. Some physiological correlates to regular and irregular phonation at the end of an utterance. $J$ Voice. 2006; 20(2):171-86.

34. Roussel NC, Lobdell M. The clinical utility of the soft phonation index. Clin Linguist Phonet. 2006; 20(2/3):181-6.

DOI: 10.1590/S1516-18462010005000002

RECEBIDO EM: 03/06/2009

ACEITO EM: 19/10/2009

Endereço para correspondência:

Giseane Conterno

Rua Presidente Kenedy, 625

Frederico Westphalen - RS

CEP: 98400-000

E-mail: gisifono@yahoo.com.br 